\title{
The optimal number of lymph nodes examined in stage II colorectal cancer and its impact of on outcomes
}

\author{
Hok Kwok Choi, Wai Lun Law* and Jensen TC Poon
}

\begin{abstract}
Background: Lymph node status is the most important prognostic factor for colorectal cancer. The number of lymph nodes that should be histologically examined has been controversial. The aims of this study were to assess the impact of the number of lymph nodes examined on survival of patients with stage II colorectal cancer and to determine the optimal number of lymph nodes that should be examined.
\end{abstract}

Methods: The study included 664 patients who underwent resection for stage II colorectal cancer. The clinical and histopathologic data of the patients were prospectively collected and analyzed.

Results: The median number of lymph nodes examined was 12 (range: 1 to 58). The 5-year disease free survival rate was significantly higher for patients with 12 or more lymph nodes examined compared to those with less than 12 lymph nodes examined. The significant difference in 5-year disease free survival persisted if the dividing number increased progressively from 12 to 23 . However, the difference in survival was most significant (lowest $p$ value and highest hazard ratio) for the number 21. The 5 -year disease free survival of patients with 21 or more lymph nodes examined was $80 \%$ whereas that of patients with less than 21 lymph nodes examined was $60 \%$ ( $p=0.001$, hazard ratio 2.08). Multivariate analysis showed that 21 or more lymph nodes examined was a factor that independently influenced survival. The 5 -year disease free survival also increased progressively with the number of lymph node examined up to the number 21. After the number 21, the survival rate did not increase further. It was likely that 21 was the optimal number, at and above which the chance of lymph node metastasis was minimal.

Conclusions: The number of lymph nodes examined in colorectal cancer specimen significantly influences survival. It is recommended that at least 21 lymph nodes should be examined for accurate diagnosis of stage II colorectal cancer.

\section{Background}

Accurate assessment for the presence of lymph node metastasis is critical in predicting the clinical outcome of patients who have undergone radical surgery for colorectal cancer. The status of the lymph nodes also largely determines whether adjuvant chemotherapy should be given; such adjuvant chemotherapy has been shown unequivocally to provide disease-free as well as overall survival benefits in patients with node positive disease [1]. However, there is always a risk of disease understaging if the extent of lymph nodes assessment is sub-optimal. A tumor with lymph node involvement may be

* Correspondence: lawwl@hkucc.hku.hk

1 Department of Surgery, The University of Hong Kong, Queen Mary Hospital, Pokfulam Road, Hong Kong

Full list of author information is available at the end of the article incorrectly classified as stage I or II if the number of lymph nodes examined is too small. The minimum number of lymph nodes that should be histologically examined for accurate staging of colorectal cancer has been controversial. While current guideline from the American Joint Committee on Cancer patient recommends the assessment of 12 lymph nodes or more [2], the recommendations in the literature range from 6 to 18 nodes [312]. The aims of this study were to assess the impact of the number of lymph nodes examined on survival of patients who underwent radical surgery for stage II colorectal cancer and to determine the optimal number of lymph nodes that should be examined. 


\section{Methods}

The study is a retrospective review and the protocol of 'Evaluation of clinical and pathological factors affecting outcomes following colorectal surgery' was approved by the Institution Review Board of the hospital. Between 2000 and 2006, a total of 664 patients underwent radical resection of colorectal cancer in the Department of Surgery, Queen Mary Hospital, The University of Hong Kong and their tumors were classified as stage II (T3-4N0M0) according to the $6^{\text {th }}$ edition of the TNM staging system of the International Union Against Cancer [13]. The clinical and histopathologic data of each patient were prospectively collected and entered into a computer database. The histopathologic features that were recorded included depth of tumor invasion (T3 or T4), tumor type (adenocarcinoma or mucinous carcinoma), tumor grade (well, moderately or poorly differentiated), lymphovascular permeation and number of lymph nodes examined.

Concerning examination of the surgical specimens, most of the specimens were fixed in $10 \%$ formalin and all were routinely processed for paraffin embedding. Lymph nodes in the specimen were identified by sight and palpation. Routine histopathologic examination was carried out using hematoxylin and eosin staining. No special fat clearance or staining techniques were routinely employed.

Adjuvant chemotherapy was given to patients with risk factors including emergency operation, mucinous carcinoma, lymphovascular permeation and less than 12 lymph nodes examined. All the patients were regularly followed up: every 3 months for the first 2 years, every 6 months for the following 3 years and then once a year. The median follow-up period was 44 months (range 12 104 months). Serum carcinoembryonic antigen level was measured regularly and imaging studies were performed in situations where recurrences were suspected.

The relationship between various clinical and histological variables and survival was evaluated using the KaplanMeier method. Differences between survival curves were tested for statistical significance by using log rank test. The Cox proportional hazard regression model was used to identify the variables that could independently influence survival. Concerning the number of lymph nodes examined, attempts were made to determine if there was a specific number at and above which the chance of lymph node involvement was minimal. Potential associations between the number of lymph nodes examined and other variables were also evaluated. Statistical Package for the Social Sciences, version 16.0 (SPSS Inc., Chicago, IL, USA) was used for all the statistical analysis. A $p$ value of less than 0.05 was considered to be statistically significant.

\section{Results}

Of the 664 patients included, 385 were men and 279 women. Their age ranged from 27 to 96 years with a mean of 70 years. Location of tumors, urgency of operation (elective or emergency), depth of tumor invasion, tumor type, tumor grade and lymphovascular permeation were summarized in Table 1 . The number of lymph nodes examined in each specimen ranged from 1 to 58 with a median of 12. The overall disease free survival of this group of patients was shown in Figure 1. The 5-year disease free survival rates in relationship to different clinical and histological variables were shown in Table 2. The 5year disease free survival rate was significantly higher in patients underwent elective operation and in patients

Table 1: Clinical and Histopathologic Data of All Patients

Number of Patients

Location of tumors

Ascending colon

131

Transverse colon

81

Descending colon

49

Sigmoid

161

Rectosigmoid junction

50

Rectum

Urgency of operation

Elective

Emergency

101

Depth of tumor invasion

T3

T4

79

Tumor type

Adenocarcinoma

Mucinous carcinoma

58

Tumor grade

$\begin{array}{ll}\text { Well differentiated } & 40 \\ \text { Moderately differentiated } & 580 \\ & \end{array}$

Lymphovascular permeation

No

576

Yes

88 


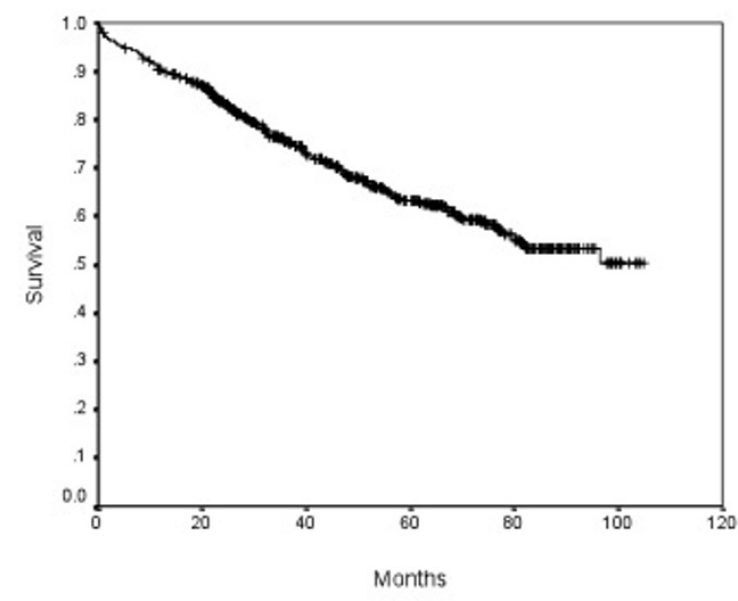

Figure 1 Disease free survival of all the 664 patients

with T3 tumor. Mucinous carcinoma and tumors with lymphovascular permeation were associated with a significantly lower survival rate.

The relationships between survival and different number of lymph nodes examined were evaluated. The 5-year disease free survival rate was significantly higher for patients with 12 or more lymph nodes examined compared to those with less than 12 lymph nodes examined. The significant difference in 5-year disease free survival persisted if the dividing number increased progressively from 12 to 23. However, the difference in survival was most significant (lowest $p$ value and highest hazard ratio) for the number 21. (Table 3) The 5-year disease free survival rate of patients with 21 or more lymph nodes examined was $80 \%$ whereas that of patients with less than 21 lymph nodes examined was $60 \%$ ( $p=0.001$, hazard ratio 2.08). (Figure 2) The 5-year disease free survival also increased progressively with the number of lymph node examined up to the number 21. After the number 21, the survival rate did not increase further. It was likely that 21 was the optimal number, at and above which the chance of lymph node metastasis was minimal. (Table 3 )

The variables including urgency of operation, depth of tumor invasion, tumor type, lymphovascular permeation and 21 or more lymph nodes examined were put into Cox proportional hazard regression model. The results showed that all the variables except tumor type were independent factors that significantly influenced survival. (Table 4)

The relationships between the number of lymph nodes examined and the other clinical and histological variables were shown in Table 5. Statistical analysis revealed that no specific variable had significant association with the number of lymph nodes examined. The number of lymph nodes examined in colon cancer specimen was also not
Table 2: 5-year Disease Free Survival Rates in Relationship to Different Clinical and Histopathologic Variables

\begin{tabular}{|c|c|c|}
\hline & $\begin{array}{l}\text { 5-year Disease } \\
\text { Free Survival }\end{array}$ & $\mathbf{p}$ \\
\hline \multicolumn{3}{|l|}{ Sex } \\
\hline Male & $60 \%$ & 0.103 \\
\hline Female & $66 \%$ & \\
\hline \multicolumn{3}{|l|}{ Tumor Location } \\
\hline Proximal to splenic flexure & $64 \%$ & 0.364 \\
\hline Distal to splenic flexure & $62 \%$ & \\
\hline \multicolumn{3}{|l|}{ Urgency of operation } \\
\hline Elective & $65 \%$ & 0.002 \\
\hline Emergency & $50 \%$ & \\
\hline \multicolumn{3}{|l|}{ Depth of tumor invasion } \\
\hline T3 & $65 \%$ & 0.000 \\
\hline $\mathrm{T} 4$ & $46 \%$ & \\
\hline \multicolumn{3}{|l|}{ Tumor type } \\
\hline Adenocarcinoma & $70 \%$ & 0.007 \\
\hline Mucinous carcinoma & $61 \%$ & \\
\hline \multicolumn{3}{|l|}{ Tumor grade } \\
\hline $\begin{array}{l}\text { Well or moderately } \\
\text { differentiated }\end{array}$ & $63 \%$ & 0.438 \\
\hline Poorly differentiated & $61 \%$ & \\
\hline \multicolumn{3}{|l|}{ Lymphovascular permeation } \\
\hline No & $65 \%$ & 0.000 \\
\hline Yes & $45 \%$ & \\
\hline
\end{tabular}

statistically different from that of rectal cancer specimen (12.9 vs. $11.3 p=0.1$ ).

\section{Discussion}

Lymph node involvement is the most important prognostic factor after radical surgery for colorectal cancer $[14,15]$. An accurate examination of the surgical specimen is mandatory to assess the lymph node status of the tumor correctly. Ideally all the lymph nodes should be harvested from the surgical specimen and examined in order to confirm that a tumor is node negative. At present, however, this goal is not practical. The actual number of lymph nodes that must be examined in the resected specimen has not yet been determined definitely. In the 
Table 3: 5-year Disease Free Survival Rates in Relationship to Number of Lymph Nodes Examined

\begin{tabular}{|c|c|c|c|c|c|}
\hline Number (N) & $\begin{array}{l}\text { 5-year disease free survival if } \\
\text { lymph nodes examined }<N\end{array}$ & $\begin{array}{l}\text { 5-year disease free survival if } \\
\text { lymph nodes examined } \geq N\end{array}$ & P Value & Hazard ratio & $\begin{array}{l}\text { 95\% confidence } \\
\text { interval }\end{array}$ \\
\hline 8 & $56 \%$ & $65 \%$ & 0.281 & 1.18 & $0.89-1.55$ \\
\hline 9 & $57 \%$ & $65 \%$ & 0.322 & 1.14 & $0.86-1.49$ \\
\hline 10 & $56 \%$ & $66 \%$ & 0.055 & 1.29 & $0.99-1.69$ \\
\hline 11 & $58 \%$ & $67 \%$ & 0.074 & 1.26 & $0.97-1.64$ \\
\hline 12 & $57 \%$ & $70 \%$ & 0.015 & 1.39 & $1.06-1.82$ \\
\hline 13 & $57 \%$ & $70 \%$ & 0.006 & 1.46 & $1.11-1.93$ \\
\hline 14 & $57 \%$ & $71 \%$ & 0.005 & 1.49 & $1.12-1.98$ \\
\hline 15 & $59 \%$ & $71 \%$ & 0.011 & 1.46 & $1.08-1.97$ \\
\hline 16 & $59 \%$ & $73 \%$ & 0.004 & 1.61 & $1.67-2.22$ \\
\hline 17 & $59 \%$ & $75 \%$ & 0.003 & 1.72 & $1.21-2.44$ \\
\hline 18 & $59 \%$ & $77 \%$ & 0.003 & 1.93 & $1.31-2.84$ \\
\hline 19 & $59 \%$ & $78 \%$ & 0.002 & 1.99 & $1.32-2.99$ \\
\hline 20 & $59 \%$ & $79 \%$ & 0.002 & 2.05 & $1.33-3.16$ \\
\hline 21 & $60 \%$ & $80 \%$ & 0.001 & 2.08 & $1.36-3.33$ \\
\hline 22 & $60 \%$ & $79 \%$ & 0.006 & 1.92 & $1.19-3.12$ \\
\hline 23 & $61 \%$ & $75 \%$ & 0.045 & 1.64 & $1.00-2.69$ \\
\hline 24 & $61 \%$ & $75 \%$ & 0.058 & 1.71 & $0.99-2.93$ \\
\hline 25 & $61 \%$ & $74 \%$ & 0.085 & 1.65 & $0.92-2.95$ \\
\hline
\end{tabular}

literature there is a lack of agreement in determining a universally valid minimum number of lymph nodes, above which there is no risk of understaging. Scott et al studied 50 cases of Dukes' $\mathrm{C}$ tumor and showed that 13 lymph nodes had to be examined to identify $94 \%$ of the specimen containing nodal metastases [3]. In 1990, the Working Party Report to the World Congress of Gastroenterology in Sydney recommended that a minimum of

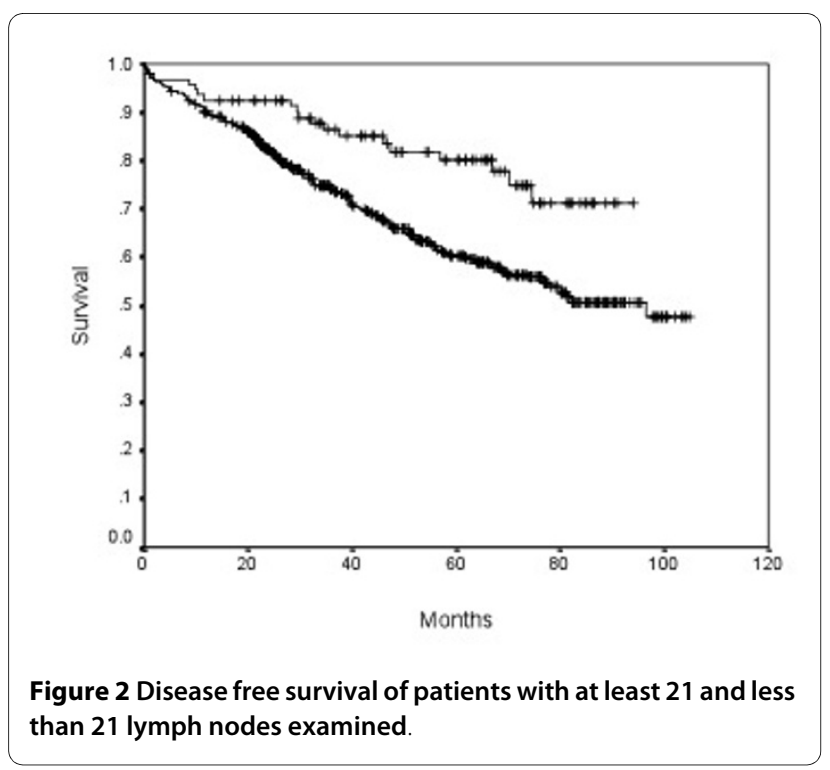

12 lymph nodes should be examined [4]. Hernanz et al, based on a group of patients with colorectal cancer in which 75 were classified as Dukes' $C$, showed that if 6 lymph nodes were examined the probability to find at least a positive lymph node was 95 percent. The probability increased to 99 percent if 10 lymph nodes were examined. The authors concluded that 6 lymph nodes provided an accurate assessment of the presence of nodal metastasis and an optimal Dukes' B classification [5]. Goldstein et al and Maurel et al showed that the probability of correctly classifying a colorectal tumor as node positive increased with the number of examined lymph nodes. They also reported that this increase had a plateau. In their two series of patients, this plateau was reached when 17 lymph nodes in one and 16 in the other had been examined [6,7]. Tepper et al divided patients with stage II rectal cancer into four quartiles according to the number of lymph nodes examined and suggested that 14 lymph nodes needed to be studied to define nodal status accurately [8]. Cianchi et al found that the 5-year survival rate of stage II patients with eight or fewer lymph nodes examined was similar to that of stage III patients. Their results suggested that examining a minimum of nine lymph nodes per surgical specimen might be sufficient for reliable staging of lymph node negative tumors [9]. Swanson et al classified patients with T3NO colon cancer into three groups according to the number of 
Table 4: Multivariate Analysis of Variables Showing Significant Association with Disease Free Survival

\begin{tabular}{llll}
\hline & Hazard Ratio & 95\% Confidence Interval & p \\
\hline Urgency of operation & & & 0.0025 \\
Depth of tumor invasion & 1.68 & $1.20-2.36$ & 0.0006 \\
Tumor type & 1.84 & $1.30-2.60$ & 0.0617 \\
Lymphovascular permeation & 1.98 & $0.96-3.96$ & 0.0001 \\
Lymph nodes examined $\geq 21$ & 2.09 & $1.48-2.96$ & 0.0025 \\
\hline
\end{tabular}

lymph nodes examined and found that a minimum of 13 lymph nodes should be examined to label the cancer as node negative [10]. Tsai et al reviewed a group of patients with T2-4NOM0 colorectal cancer and suggested that examining a minimum of 18 lymph nodes per surgical specimen might be taken into consideration for more reliable staging of lymph node negative cancer [11].
Vather et al analyzed various lymph node strata and showed a sharp and statistically significant drop in recurrence rate after the $16^{\text {th }}$ node mark. The recurrence rate remained at a low level for the remaining strata [12]. In our study, we demonstrated that the number of lymph nodes examined significantly influenced survival and the difference in survival was most significant when compar-

Table 5: Number of Lymph Nodes Examined in Relationship to Different Clinical and Histopathologic Variables

\begin{tabular}{|c|c|c|}
\hline & Number of lymph nodes & $P$ Value \\
\hline \multicolumn{3}{|l|}{ Sex } \\
\hline Male & 11.84 & 0.168 \\
\hline Female & 12.70 & \\
\hline \multicolumn{3}{|l|}{ Tumor Location } \\
\hline Proximal to splenic flexure & 12.89 & 0.654 \\
\hline Distal to splenic flexure & 12.34 & \\
\hline \multicolumn{3}{|l|}{ Urgency of operation } \\
\hline Elective & 12.08 & 0.379 \\
\hline Emergency & 12.84 & \\
\hline \multicolumn{3}{|l|}{ Depth of tumor invasion } \\
\hline $\mathrm{T} 3$ & 12.36 & 0.136 \\
\hline $\mathrm{T} 4$ & 10.93 & \\
\hline \multicolumn{3}{|l|}{ Tumor type } \\
\hline Adenocarcinoma & 12.04 & 0.082 \\
\hline Mucinous carcinoma & 13.98 & \\
\hline \multicolumn{3}{|l|}{ Tumor grade } \\
\hline Well or moderately differentiated & 12.12 & 0.395 \\
\hline Poorly differentiated & 13.10 & \\
\hline \multicolumn{3}{|l|}{ Lymphovascular permeation } \\
\hline No & 12.21 & 0.907 \\
\hline Yes & 12.10 & \\
\hline
\end{tabular}


ing patients with 21 or more lymph nodes and those with less than 21 lymph nodes examined. Indeed, 21 or more lymph nodes examined was an independent prognostic factor associated with better disease free survival. We also found that the 5-year disease free survival rate increased progressively with the number of lymph node examined up to the number 21. After the number 21, the survival rate did not increase further. It was likely that 21 was the optimal number, at and above which the chance of lymph node metastasis was minimal. We recommend that a minimum of 21 lymph nodes should be examined to label a tumor as node negative. The variability in searching ability for lymph nodes by pathologists and the different statistical methods employed are probably the major factors that explain the considerable variation among the different studies.

A proportion of patients in our study had adjuvant chemotherapy administered. Ideally this group of patients should be excluded when the survival rates were evaluated. However, in our practice adjuvant chemotherapy was only given to stage II patients with risk factors including emergency operation, mucinous carcinoma, lymphovascular permeation and less than 12 lymph nodes examined. These factors were all shown to be associated with lower survival rates. Therefore the inclusion of patients treated with chemotherapy should not affect the overall results.

The number of lymph nodes recovered from resection specimens is dependent on several factors. The variability in the number of lymph nodes in various regions of the large bowel and the extent of surgical lymphadenectomy alter the exact number of lymph nodes in a resection specimen. The diligence and skill of the pathologist in identifying and harvesting lymph nodes in the specimen determine the actual number of lymph nodes examined. It has been shown that nodal metastasis in colorectal cancer is often found in small lymph nodes $(<5 \mathrm{~mm}$ in diameter) [16,17], diligent search for lymph nodes is required on gross examination of resection specimens. Of note, many pathologists are uninformed about the necessity of examining a critical number of lymph nodes to accurately stage colorectal cancers. One Canadian study showed that only $58 \%$ of pathologists were aware of guidelines for lymph node retrieval in colorectal cancer at all and that as few as $25 \%$ knew that a minimum of 12 lymph nodes (National Cancer Institute guidelines 2000) is necessary for accurate designation of node negativity [18].

Lymph node collection from colorectal resection specimen is time consuming, particularly if the lymph nodes are small. Small lymph nodes are difficult to be found, especially amid large amount of mesenteric fat. Owing to the lack of widely accepted pathology practice standards for lymph node examination in colorectal cancer specimens, there are many variations in the basic pathologic techniques used for lymph node collection and submis- sion for microscopic analysis. Some of these variations include the use of clearing solutions to improve visualization of small lymph nodes in the pericolonic or perirectal fat, the submission of one half versus both halves of each node for microscopic examination, and the preparation of one versus more than one tissue level per paraffin block of submitted nodal tissue. In an effort to reduce this variation, the College of American Pathologists has recommended that all grossly negative or equivocal lymph nodes be submitted in their entirety for microscopic examination. For grossly positive lymph nodes, it is recommended that a representative sample be submitted for microscopic confirmation. However, if fewer than 12 lymph nodes are found after careful gross examination, it is suggested that additional visual enhancement techniques that aid in the macroscopic identification of lymph node, such as fat clearing, be considered $[3,19]$. Unfortunately, the purchase and disposal costs for chemicals required for these techniques are high.

Hsu et al demonstrated that larger tumor, (tumor localization) and (depth of tumor invasion) were associated with a higher number (12 or more) of harvested lymph nodes in colorectal cancer specimen [20]. On the other hand, Horzic et al found that male gender, greater tumor size, better tumor differentiation as well as presence of acute inflammation in the abdominal cavity were independent predictors of increased number of examined lymph nodes [21]. According to the author, well-differentiated tumors were associated with increased number of examined lymph nodes. That might be due to better immune response against better-differentiated tumors. The association between acute inflammation in the abdominal cavity and number of lymph nodes examined might be explained by the fact that any inflammation causes reactive hyperplasia of lymph nodes. In our study, however, we could not identify any clinical or histological factor that influenced the number of lymph nodes examined.

Instead of exhaustive lymph node harvest and examination, an alternative way to determine lymph node status is sentinel lymph node biopsy. This approach has been extensively used for breast cancer and melanoma. However, the value of sentinel lymph node biopsy for colorectal cancer is still limited. A lot of studies have evaluated the role of sentinel lymph node biopsy in the management of colorectal cancer. At present sentinel lymph node biopsy has not been shown to be a reliable predictor of No status due to its relatively high false negative rate $[22,23]$. Formal lymphadenectomy and intensive lymph node examination are still essential for colorectal cancer.

\section{Conclusions}

In summary, the number of lymph nodes examined in colorectal cancer specimen significantly influenced survival and the difference in survival was most significant 
when comparing patients with 21 or more lymph nodes and those with less than 21 lymph nodes examined. Twenty one or more lymph nodes examined was an independent prognostic factor associated with better disease free survival. The chance of lymph node metastasis was likely minimal if at least 21 lymph nodes showed no tumor involvement. We thus recommend that a minimum of 21 lymph nodes should be examined to label a tumor as node negative. The diligence and skill of pathologists remains an important factor that determines the actual number of lymph node examined. It is unlikely that sentinel lymph node biopsy can substitute for intensive lymph node examination in the management of colorectal cancer.

\section{Competing interests}

The authors declare that they have no competing interests.

\section{Authors' contributions}

WLL conceived of the study. HKC designed the study, performed the statistical analysis and drafted the manuscript. WLL revised the manuscript. All authors read and approved the final manuscript.

\section{Author Details}

Department of Surgery, The University of Hong Kong, Queen Mary Hospital, Pokfulam Road, Hong Kong

Received: 30 August 2009 Accepted: 8 June 2010

Published: 8 June 2010

\section{References}

1. Wolmark N, Rockette H, Fisher B, Wickerham DL, Redmond C, Fisher ER, Jones J, Mamounas EP, Ore L, Petrelli NJ: The benefits of leucovorin modulated fluorouracil as postoperative therapy for primary colon cancers: results from the National Surgical Adjuvant Breast and Bowel Project. J Clin Oncol 1993, 11:1879-87.

2. Nelson H, Petrelli N, Carlin A, Couture J, Fleshman J, Guillem J, Miedema B, Ota D, Sargent D: National Cancer Institute Expert Panel. Guidelines 2000 for colon and rectal cancer surgery. J Natl Cancer Inst 2001, 93:583-96.

3. Scott KWM, Grace RH: Detection of lymph node metastases in colorectal cancinoma before and after fat clearance. Br J Surg 1989, 76:1165-7.

4. Fielding LP, Arsenault PA, Chapuis PH, Dent O, Gathright B, Hardcastle JD, Hermanek P, Jass JR, Newland RC: Clinicopathologic staging for colorectal cancer: an international documentation system (IDS) and an international comprehensive anatomical terminology (ICAT). J Gastroenterol Hepatol 1991, 6:325-44.

5. Hernanz F, Revuelta S, Redondo C, Madrazo C, Castillo J, Gómez-Fleitas M: Colorectal adenocarcnoma: quality of the assessment of lymph node metastases. Dis Colon Rectum 1994, 37:373-7.

6. Goldstein NS, Sanford W, Coffey M, Layfield L: Lymph node recovery from colorectal resection specimens removed for adenocarcinoma: trends over time and a recommendation for a minimum number of lymph nodes to be recovered. Am J Clin Pathol 1996, 106:209-16.

7. Maurel J, Launoy G, Grosclaude P, Gignoux M, Arveux P, Mathieu-Daudé $H$, Raverdy N, Faivre J: Lymph node harvest reporting in patients with carcinoma of the large bowel: a French population-based study. Cancer 1998, 82:1482-6.

8. Tepper JE, O'Connell MJ, Niedzwiecki D, Hollis D, Compton C, Benson AB, Cummings B, Gunderson L, Macdonald JS, Mayer RJ: Impact of number of nodes retrieved on outcome in patients with rectal cancer. J Clin Oncol 2001, 19:157-63.

9. Cianchi F, Palomba A, Boddi V, Messerini L, Pucciani F, Perigli G, Bechi P, Cortesini C: Lymph node recovery from colorectal tumour specimens: Recommendation for a minimum number of lymph nodes to be examined. World J Surg 2002, 26:384-9.
10. Swanson RS, Compton CC, Stewart AK, Bland KI: The prognosis of T3NO colon cancer is dependent on the number of lymph nodes examined. Ann Surg Oncol 2003, 10:65-71.

11. Tsai HL, Lu CY, Hsieh JS, Wu DC, Jan CM, Chai CY, Chu KS, Chan HM, Wang $J Y:$ The prognostic significance of total lymph node harvest in patients with T2-4NOMO colorectal cancer. J Gastrointest Surg 2007, 11:660-5.

12. Vather R, Sammour T, Zargar-Shoshtari K, Metcalf P, Connolly A, Hill A: Lymph node examination as a predictor of long term outcome in Dukes B colon cancer. Int J Colorectal Dis 2009, 24:283-8.

13. International Union Against Cancer (UICC): TNM classification of malignant tumors. 6th edition. Edited by: Sobin LH, Wittekind CH. New York: Wiley; 2002.

14. Newland RC, Chapuis PH, Smythe EJ: The prognostic value of substaging colorectal carcinoma: a prospective study of 1117 cases with standardized pathology. Cancer 1987, 60:852-7.

15. Wolmark N, Fisher B, Wieand HS: The prognostic value of the modification of the Dukes $C$ class of colorectal cancer: an analysis of the NSABP trial. Ann Surg 1986, 139:846-52.

16. Brown HG, Luckasevic TM, Medich DS, Celebrezze JP, Jones SM: Efficacy of manual dissection of lymph nodes in colon cancer resections. Mod Pathol 2004, 17:402-6.

17. Herrera-Ornelas L, Justiniano J, Castillo N, Petrelli NJ, Stulc JP, Mittelman A: Metastases in small lymph nodes from colon cancer. Arch Surg 1987, 122:1253-6.

18. Wright FC, Law CH, Last LD, Ritacco R, Kumar D, Hsieh E, Khalifa M, Smith $\mathrm{AJ}$ : Barriers to optimal assessment of lymph nodes in colorectal cancer specimens. Am J Clin Pathol 2004, 121:663-70

19. Compton C, Fielding L, Burgart L, Conley B, Cooper HS, Hamilton SR, Hammond ME, Henson DE, Hutter RV, Nagle RB, Nielsen ML, Sargent DJ, Taylor CR, Welton M, Willett C: Prognostic factors in colorectal cancer: College of American Pathologists consensus statement 1999. Arch Pathol Lab Med 2000, 124:979-94.

20. Hsu CW, Lin CH, Wang HT, Ou WC, King TM: Factors that influence 12 or more harbested lymph nodes in early-stage colorectal cancer. World J Surg 2009, 33:333-9.

21. Horzic M, Kopljar M: Minimal number of lymph nodes that need to be examined for adequate staging of colorectal cancer - Factors influencing lymph node harvest. Hepatogastroenterology 2005, 52:86-9.

22. Bembenek AE, Rosenberg R, Wagle E, Gretschel S, Sendler A, Siewert JR, Nährig J, Witzigmann H, Hauss J, Knorr C, Dimmler A, Gröne J, Buhr HJ, Haier J, Herbst H, Tepel J, Siphos B, Kleespies A, Koenigsrainer A, Stoecklein NH, Horstmann O, Grützmann R, Imdahl A, Svoboda D, Wittekind C, Schneider W, Wernecke KD, Schlag PM: Sentinel lymph node biopsy in colon cancer: a prospective multicenter trial. Ann Surg 2007, 245:858-63

23. Quadros CA, Lopes A, Araujo I, Fregnani JH, Fahel F: Upstaging benefits and accuracy of sentinel lymph node mapping in colorectal adenocarcinoma nodal staging. J Surg Oncol 2008, 98:324-30.

Pre-publication history

The pre-publication history for this paper can be accessed here: http://www.biomedcentral.com/1471-2407/10/267/prepub

doi: $10.1186 / 1471-2407-10-267$

Cite this article as: Choi et al., The optimal number of lymph nodes examined in stage II colorectal cancer and its impact of on outcomes BMC Cancer 2010, 10:267 